\title{
SEASONAL VARIATION IN SLIPPED CAPITAL FEMORAL EPIPHYSIS: NEW FINDINGS USING A NATIONAL CHILDREN'S HOSPITAL DATA BASE
}

\author{
Randall T. Loder, MD \\ Chris A. Schneble, BS
}

From the Department of Orthopaedic Surgery, Indiana University School of Medicine

James Whitcomb Riley Children's Hospital, Indianapolis, Indiana, 46202 USA

This research was supported in part by the Garceau Professorship Endowment, Indiana University, School of Medicine, Department of Orthopaedic Surgery, and the Rapp Pediatric Orthopaedic Research Endowment, Riley Children's Foundation, Indianapolis, Indiana.

Address all correspondence and requests for reprints to

Randall T. Loder, MD

James Whitcomb Riley Hospital for Children

705 Riley Hospital Drive, ROC 4250

Indianapolis, Indiana 46202

317-948-0961

FAX 317-944-7120

rloder@iupui.edu

This is the author's manuscript of the article published in final edited form as:

Loder, R., \& Schneble, C. (2019). Seasonal Variation in Slipped Capital Femoral Epiphysis: New Findings Using a National Children's Hospital Database. Journal of Pediatric Orthopaedics, 39(1). https://doi.org/10.1097/BPO.0000000000001074 
ABSTRACT

Background: Slipped capital femoral epiphysis (SCFE) demonstrates seasonal variation in certain latitudes but not others. Is such variation influenced by temperature differences, sunlight exposure and subsequent vitamin D production or other climate variables? It was the purpose of this study to further investigate the seasonal variation in 10 month of presentation for SCFE.

11 Methods: Data for this study originated from the Pediatric Hospital Information System (PHIS) for all children 12 with a diagnosis of SCFE from January 1, 2004 through December 31, 2014. From this database the patient's 13 gender, ethnicity, hospital location, and month of presentation was determined. Only those patients treated primarily 14 for SCFE were included. Geographic and climate data (latitude, average annual temperature, precipitation, climate 15 type [Köppen-Geiger and Liss], horticultural plant zone hardiness, and sunlight exposure) for each of the 49 PHIS 16 hospitals was determined. Seasonal variation was analyzed using cosinor analysis. A p <0.05 was considered 17 statistically significant.

18 Results: There were 10,350 cases of SCFE with an overall peak presentation in mid August. For those living at a 19 latitude of $>35^{\circ} \mathrm{N}$ there was single peak, a less prominent double peak for those $31-35^{\circ} \mathrm{N}$, and no variation for 20 those $<31^{\circ} \mathrm{N}$. As the average annual temperature increased there was less seasonal variability. Humid, temperate 21 and cold winter climates demonstrated seasonal variation while other climate types did not. Those living in areas 22 having < 2500 hours of sunlight per year demonstrated seasonal variation. Further, areas having a photovoltaic solar 23 production potential $<5.0 \mathrm{kWh} / \mathrm{m}^{2} /$ day also demonstrated seasonal variation.

24 Conclusions: We discovered new seasonal variation findings regarding SCFE. These are a double peak pattern for 25 those between $31-35^{\circ} \mathrm{N}$ latitude; less variability as the average annual temperature increases; and sunlight exposure 26 correlates with seasonal variability. Potential explanations are a rachitic state due to seasonal variation in vitamin D 27 production, and seasonal variation in physeal growth and strength. These new findings will require further 28 investigation. 
Slipped capital femoral epiphysis (SCFE) often demonstrates seasonal variation in both presentation to the clinician and onset of the disorder (1-4). Many questions have arisen from these studies, especially differences between varying degrees of latitude $(3,5)$ - how can that be explained? Does such variation represent temperature differences, sunlight exposure with different amounts vitamin D production (6), or other even more esoteric climactic variables? With the advent of large national data bases, seasonal variation in SCFE, if it does exist, can be more fully explored. It was the purpose of this study to further investigate the seasonal variation in SCFE.

\section{MATERIALS AND METHODS}

Data for this study was obtained from the Pediatric Hospital Information System (PHIS), an administrative database that contains inpatient, emergency department, ambulatory surgery and observation encounter-level data from over 45 not-for-profit, tertiary care pediatric hospitals in the United States which are affiliated with the Children's Hospital Association (Overland Park, KS). This data base is being increasingly used in pediatric studies with over 500 published peer reviewed manuscripts as of July 2017 (personal communication, Mr. Shawn Reid, Children's Hospital Association analytics). Data quality and reliability are assured through a joint effort between the Children's Hospital Association and participating hospitals. Portions of the data submission and data quality processes for the PHIS database are managed by Truven Health Analytics (Ann Arbor, MI). Data is de-identified and subjected to a number of reliability and validity checks before included in the database. Although primarily a financial administrative database, there is large amount of clinical information such as demographics, episodes of care, and treatment(s) rendered. The study was determined to be exempt by our local Institutional Review Board.

The data base was queried for those children with a primary ICD 9 diagnosis of 732.2 (nontraumatic slipped upper femoral epiphysis) from January 1, 2004 through December 31, 2014. The following information was obtained: gender and ethnicity, hospital, medical record number, date of birth and admission, and treatment rendered. Treatment for non SCFE related issues was excluded (e.g. emergency room visits for asthma, etc.). We only included those patients treated with internal fixation in-situ, closed reduction and internal fixation, and open reduction and internal fixation. Procedures such as osteotomy, osteoplasty, etc. were excluded as they were likely reconstructive procedures and most likely not the first SCFE treatment. Cases due to complications associated with SCFE (e.g. fracture, avascular necrosis, complications with internal fixation, infection, etc.) were excluded as well as endocrine or renal associated SCFEs.

The month of presentation for the SCFE was defined as the initial month that each particular patient was entered into the data base for the initial SCFE treatment episode. When there was more than one month per patient, each treatment was reviewed to ensure that the subsequent procedures were not for reconstructive procedures or those related to a complication. If not, then the $2^{\text {nd }}$ presentation was considered to be the opposite hip in a child with sequential presentations of bilateral SCFE. Thus sequential bilateral SCFEs are counted twice, while any child having a simultaneous bilateral SCFE presentation is counted only once. The PHIS does not record the duration of symptoms making it impossible to determine the month of onset.

Geographic and climate data were collected for each of the 49 PHIS hospital cities (Supplemental Table 1) from the National Oceanic and Atmospheric Administration (7). We collected several measures of climate severity and sunlight exposure. These were 1) horticultural plant zone hardiness, 2) climate type $(8,9), 3)$ cumulative sun 
exposure in hours per year (10), and 4) potential solar electrical production from photovoltaic resources (11) (Supplemental Figures 1-3).

The horticultural plant zone hardiness scale (12) is one proxy for climate severity. The zones vary from 1 to 13 and represent the average annual minimum temperature with 1 being the coldest; it can be accessed at http://planthardiness.ars.usda.gov. Climate type was categorized using both the well known Köppen-Geiger classification (8) and the newer Liss classification (9). The Köppen-Geiger classification is composed of 3 letters. The $1^{\text {st }}$ letter is a description of the main climate, the $2^{\text {nd }}$ the amount of precipitation, and the $3^{\text {rd }}$ the temperature, for 31 different climate types; it can be accessed at http://koeppen-geiger.vu-wien.ac.at. Liss et al. (9) condensed these 31 types into eight types involving a four letter scheme. The $1^{\text {st }}$ two letters represent the winter and the $2^{\text {nd }}$ two the summer; the $1^{\text {st }}$ and $3^{\text {rd }}$ letters are upper case and designate the climate type $(\mathrm{C}=$ cold, $\mathrm{H}=$ hot, $\mathrm{T}=$ temperate $)$ while the $2^{\text {nd }}$ and $4^{\text {th }}$ are lower case and designate the amount of precipitation $(\mathrm{a}=$ arid, $\mathrm{d}=$ moderately dry, and $\mathrm{w}=$ wet $)$. For example, TwCd is a climate with a temperate, wet winter and a cold, moderately dry summer.

Sunlight exposure for each PHIS hospital was quantified using two different methods. The first was hours of sunlight exposure each year (10). The second more exact method used photovoltaic solar resource, a measure of the ability to transform sunlight energy into other products, such as electricity or vitamin D. Each city was grouped by $0.5 \mathrm{kWh} / \mathrm{m}^{2} /$ day increments, using National Renewal Energy Laboratory data, and can be accessed at http://www.nrel.gov/gis/solar.html. (11).

\section{Statistical Analysis}

Univariate and bivariate analyses were used to determine the mean and standard deviation for continuous variables and frequencies/percentages for categorical variables. Temporal variation was analyzed using cosinor analysis (13) which represents a mathematical best fit of the data to a curve defined by the equation $\mathrm{F}(\mathrm{t})=\mathrm{M}+$ $\mathrm{A} \cos (\omega \mathrm{t}+\phi)$, where $\mathrm{M}=$ the mean level (termed mesor), $\mathrm{A}=$ the amplitude of the cosine curve, $\phi=$ acrophase (phase angle of the maximum value), $\omega=$ the frequency ( which for monthly analysis is $360^{\circ} / 12=30^{\circ}$ ), and $\mathrm{t}=$ time (which in this case is each month). The overall $\mathrm{p}$ and $\mathrm{r}^{2}$ values represent a rhythmic pattern described by the cosinor equation for $\mathrm{M}, \mathrm{A}$, and $\phi$. The data was analyzed for the entire period of 12 months, as well as decreasing increments of 1 month. A best fit may not be a period of 12 months, but a different time span (e.g. 6 months periodicity). Cosinor analyses were performed with ChronoLab 3.0 ${ }^{\mathrm{TM}}$ software (Acknowledgement). All other analyses were performed using Systat $10^{\mathrm{TM}}$ software (Chicago, Illinois, 2000). A p $<0.05$ was considered statistically significant for all analyses.

\section{RESULTS}

A total of 13,168 procedures were performed in 11,058 unique SCFE patients. There were 10,350 treatment episodes appropriate for seasonal analysis. Overall, there was a peak presentation in mid August (Figure 1) which did not differ by gender, Black/White ethnicity, or unilateral/bilateral nature. There were significant single peaks for those $>35^{\circ} \mathrm{N}$ latitude, a less prominent double peak for those $31-35^{\circ} \mathrm{N}$ latitude, and no variation for those $<31^{\circ} \mathrm{N}$ latitude (Figure 2). As the average annual temperature (Supplemental Table 2) or plant zone increased (Supplemental Figure 4) there was less seasonal variability. Seasonal variation was absent in arid locations but 
106

107

108

109

110

111

112

113

114

115

116

117

118

119

120

121

122

123

124

125

126

127

128

129

130

131

132

133

134

135

136

137

138

139

140

141

142 present in wetter climates. Thus latitude, ambient temperature, and precipitation correlate with SCFE month of presentation.

Analyses using the Köppen-Geiger and Liss classification schemes (Supplemental Table 3) demonstrated that single peaks occurred in temperate and cold winter climates that are relatively humid; warm arid or fully hot and humid climates demonstrated minimal variation. Sunlight exposure was also associated with seasonal variation in SCFE (Supplemental Table 4). Those living in areas having < 2500 hours of sunlight per year demonstrated seasonal variation (Supplemental Figure 5A) as well as those where the solar voltaic production was $<5.0$ $\mathrm{kWh} / \mathrm{m}^{2} /$ day (Supplemental Figure 5B).

\section{DISCUSSION}

Seasonal variation in SCFE was first described in 1971 (14). There was a July to November peak in boys, which was attributed to increased physical activity from playing football. In girls, the peak range was one month earlier. Subsequent studies have confirmed a seasonal variation in SCFE, with peak presentations in the late summer or early autumn (1-4). A USA wide study noted variations both north and south of the $40^{\circ} \mathrm{N}$ latitude; $57.4 \%$ of those north occurred April through September, while 57.3\% of those south occurred October through March (15).

We must first acknowledge potential weaknesses of this study. There is always the possibility that certain data was wrongly entered into the data base, such as the wrong diagnosis or treatment. Also, it is possible that not all cases of SCFE each year for each hospital were entered. The magnitude of such a potential error is difficult to know. However, failure to enter a case would most likely be a random event, and thus equally distributed over an entire year which would not create any biased error regarding seasonal variation. Even if only $50 \%$ of the cases were entered (eg 10,350 of 20,700), such a 50\% sample would have, at a $95 \%$ confidence level, a margin of error of only $0.68 \%$ (see Supplemental Materials for further discussion). Next, for any reader of this study working at one of the hospital locations described in Supplemental Table 1, must understand that the number of cases of SCFE at their institution over the time span of this study (2004 through 2014) may not be the actual number shown in Supplemental Table 1. This is due to two issues. The first is that not all hospitals became PHIS members in 2004. Many of the hospitals became PHIS members distributed at different points along the time line from 2004 - 2014 , which would appear to demonstrate a conflicting numbers of cases. The second is that we excluded secondary and reconstructive cases; a hospital having a high volume of quaternary referrals for osteotomies or other reconstructive procedures will have a larger number of SCFE cases than noted in this study. Another potential weakness is that we may have excluded some patients with bilateral disease at the time a second, sequential SCFE presented. However, the percentage of sequential bilaterality was $20.1 \%$ (2,083 in 10,350), very similar to other figures of bilaterality. Another potential criticism is that the latitude of where the patient lives is markedly different than the treating hospital. That could clearly be present if the treatment was referral to a quaternary care center for advanced hip preservation surgery; however, any potential reconstructive cases were excluded. It is unlikely that large number of patients traveled enough distance to change the latitude grouping by $5^{\circ}$, the latitude strata used in this study, for their initial care. Finally, the data used to determine plant hardiness zones, climate classification, and solar energy potential does not exactly span the same years as this study, but these are the most recent data available. Since we 
averaged the results over 11 years, and since the climate graphs also used averaging methods, we propose that those graphs are very applicable to our data.

The major strength of this study is that it is the largest series to date (10,350 cases) reviewing seasonal variation of SCFE, more than double the previous largest United States series of 4,690 (3). Also, the data in this study is actual patient data, not a $20 \%$ sampling of discharge data from the Nationwide Inpatient Sample used by Brown (3).

No mathematical modeling of biologic processes is perfect. A perfect fit of any data would demonstrate an $\mathrm{r}^{2}$ of 1.00. When reviewing the statistically significant cosinor models in this study, the reader should understand that a $\mathrm{p}<0.05$, although significant, may show considerable variability as demonstrated by the $\mathrm{r}^{2}$ value. The goal of this study was not to fit the data to a highly accurate, complex mathematical model, but rather to demonstrate trends that might show further insight into the etiology of SCFE. This caveat must be remembered when reviewing our results. Further details are discussed in the Supplemental Materials.

With these caveats in mind, in this study, we confirmed many of the previous findings and discovered new ones regarding SCFE seasonal variation. For those living north of the $35^{\circ} \mathrm{N}$ latitude, there was significant seasonal variation, with a peak presentation mid to late August. In the more southern latitudes, there was a double peak pattern for those between $31-35^{\circ} \mathrm{N}$ latitude and no variation for those $<31^{\circ} \mathrm{N}$ latitude. This is the first that a double peak has been described in the USA. Such a peak was noted in a nation-wide study in Japan. The latitude of Tokyo, the most populous city in Japan, is $36^{\circ} \mathrm{N}$, very similar to the $31-35^{\circ} \mathrm{N}$ in this study.

This is the first study to explore correlations between SCFE seasonal variation and climate. As the average annual temperature increases, there is less variability. Seasonal variation was absent in arid locations. There were single peaks in the relatively humid, temperate and cold winter climates. Warm arid climates or fully hot and humid climates demonstrated no significant variation. Finally, the magnitude of sunlight exposure was associated with seasonal differences. Those living in cities with $<2500$ hours of sunlight exposure per year or photovoltaic solar production potential $<5.0 \mathrm{kWh} / \mathrm{m}^{2} /$ day demonstrated seasonal variation; those having more sunlight exposure demonstrated no seasonal variation.

There are many potential explanations for these findings. An intriguing one is that SCFE may represent a rachitic state, which has been postulated by several authors $(3,5,6)$. Such seasonal variation could be explained by differences in vitamin D production and levels at different times of the year. It is well known that vitamin D levels in children vary by time of the year (16). The high prevalence of vitamin D insufficiency/deficiency in children and adolescents (16) is higher in Blacks than in Caucasians (17) and in obese compared to non obese children (18). These findings nicely fit the known demographics of SCFE which is more common in obese (19) and Black $(15,19)$ children. Vitamin D deficient rats demonstrate extensive disorganization in the growth plate (20). In such rats the chondrocyte columns in the hypertrophic and proliferative zones are aligned in varying directions and not parallel to the axis of growth, impacting its mechanical properties to shear stress. This histopathologic disarray is also seen in SCFE.

Few studies address vitamin D levels in SCFE. A study of 20 consecutive children from Los Angeles with SCFE and found no vitamin D deficiency, regardless of obesity status. The majority of the children studied were 
Hispanic (17 of 20), but the time of year when the vitamin D levels were collected was not given (21). In Vellore, India, all 15 children with SCFE had significantly lower vitamin D levels than the controls (22). In Southampton, England (23) $85 \%$ of children with SCFE were vitamin D deficient.

Another intriguing hypothesis is the relationship between seasonal variation in SCFE and seasonal variation in height and weight growth velocity. In general, maximum height velocity occurs in the spring and summer, and maximum weight velocity in the fall and winter. The physis becomes weaker with increasing physeal thickness. Does a maximum height gain in the spring and summer, with the theoretical increase in physeal height during maximum height growth, result in SCFE that then presents slightly thereafter? The correlation between physeal height and SCFE has been described in humans, with acceleration in skeletal growth just before the slip occurs (24).

The bimodal pattern noted in the more southern, warmer latitudes is intriguing. Some authors have postulated increased physical activity at the peak onset of SCFE (14). Such activity is difficult to quantify, especially in a review using this type of a data base. However, the bimodal pattern seen in the southern, warmer latitudes could be explained by more physical activity in the spring and autumn when the weather is more amenable to outdoor activity compared to the very hot summer. This bimodal pattern can not be explained by seasonal variations in vitamin $\mathrm{D}$, as studies from more southern latitudes note that vitamin $\mathrm{D}$ levels are higher in the summer and lower in the winter like that seen in more northern latitudes.

We could not analyze the month of onset of the SCFE, as the duration of symptoms was not known. Other studies addressing seasonal variation of SCFE using national databases have the same handicap of not knowing the duration of symptoms $(3,15)$. Previous studies that determined month of onset $(1,5,25)$ subtracted the symptom duration from the month of presentation. The average duration of symptoms was approximately four months in all three studies. This time interval in diagnosis of SCFE has not changed for decades and ranges from two to 6.5 months. If we assume that the average symptom duration is 3 to 4 months (some will be greater, and others less, but on average this 3 to 4 months is likely a reasonable estimate), then the overall month of onset would be around May, similar to an older study (1).

In conclusion, this is the first study to investigate other climate influences on seasonal variation in SCFE. It has corroborated many of the other studies demonstrating a seasonal variation in SCFE, yet has also given new insights into the possible effects of latitude and climate variables on seasonal variation of SCFE. The synthesis and explanations of these new findings will require further investigation. 


\section{ACKNOWLEDGEMENTS}

210 ChronoLab 3.0 ${ }^{\mathrm{TM}}$ software, designed for use on Macintosh ${ }^{\mathrm{TM}}$ computers, cannot be purchased. The software used to 211 perform cosinor analyses was provided through the courtesy of Dr. Artemio Mojón and colleagues, Bioengineering

212 and Chronobiology Labs, ETSI Telecomunicación, University of Vigo, Campus Universitario, Vigo (Pontevedra)

213 36280, Spain. It can be downloaded from their web site at http://www.tsc.uvigo.es/BIO/Bioing/References.html.

214 Please kindly acknowledge their generosity when using this software.

215 


\section{REFERENCES}

217 1. Loder RT, Aronson DD, Bollinger RO. Seasonal variation of slipped capital femoral epiphysis. J Bone 218 Joint Surg [Am] 1990;72-A:378-381.

219 2. Maffulli N, Douglas AS. Seasonal variation of slipped capital femoral epiphysis. J Pediatr Orthop B $220 \quad 2002 ; 11: 29-33$.

221 3. Brown D. Seasonal variation of slipped capital femoral epiphysis in the United States. J Pediatr Orthop $222 \quad 2004 ; 24: 139-143$.

223 4. Andrén L, Borgström K-E. Seasonal variation of epiphysiolysis of the hip and possible causative factor.

224 Acta Orthop Scand 1959;28:22-26.

225 5. Loder RT, and 47 coinvestigators from 33 orthopaedic centers and 6 continents. A worldwide study on the 226 seasonal variation of slipped capital femoral epiphysis. Clin Orthop 1996;322:28-36.

227 6. Farrier AJ, Ihediwa U, Khan S, Kumar A, Gulati V, Uzoigwe CE, et al. The seasonality of slipped upper 228 femoral epiphysis - a meta-analysis: a possible association with vitamin D. Hip Int 2015;26:495-501.

229 7. National Oceanic and Atmospheric Administration, National Centers for Environmental Information.

230 http://www.ncdc.noaa.gov/cdo-web/datasets Accessed August 24, 2016.

$231 \quad$ 8. Kottek M, Grieser J, Beck C, Rudolf B, Rubel F. World map of the Köppen-Geiger climate classification 232 updates. Meteorol Z 2006;15:259-263.

2339 - 9 . Liss A, Koch M, Naumova EN. Redefining climate regions in the United States of America using satellite 234 remote sensing and machine learning for public health applications. Geospat Health 2014;8:S647-S659.

235 10. All the Dirt on Gardening. Europe vs. the United States. Sunshine duration in hours per year.

236 http://allthedirtongardening.blogspot.com/2013/12/hours-of-sunlight-europe-and-us.html. Accessed June 12, 2016.

237 11. Roberts BJ. Photovoltaic resource of the United States.

238 http://www.nrel.gov/gis/images/eere_csp/national_concentrating_solar_2012-01.jpg. Accessed June 12, 2016.

239 12. Agricultural Research Service, U.S. Department of Agriculture, Plant Hardiness Zone Map.

240 http://planthardiness.ars.usda.gov. Accessed June 12, 2016.

241 13. Nelson W, Tong YL, Lee J-K, Halberg F. Methods for cosinor-rhythymometry. Chronobiologia $2421979 ; 6: 305-323$.

243 14. Ferguson AB, Howorth MB. Slipping of the upper femoral epiphysis. JAMA 1931;97:1867-1872.

244 15. Lehmann CL, Arons RP, Loder RT, Vitale MG. The epidemiology of slipped capital femoral epiphysis:

245 an update. J Pediatr Orthop 2006;26:286-290.

246 16. Poskitt EM, Cole TJ, Lawson DEM. Diet, sunlight, and 25-hydroxy vitamin D in healthy children and 247 adults. BMJ 1979;1:221-223.

248 17. Rajakumar K, Holick MF, Jeong K, Moore CG, Chen TC, Olabopo F, et al. Impact of season and diet on 249 vitamin D status of African-American and Caucasian children. Clin Pediatr 2011;50:493-502.

250 18. Alemzadeh R, Kichler J, Babar G, Calhoun M. Hypovitaminosis D in obese children and adolescents:

251 relationship with adiposity, insulin sensitivity, ethnicity, and season. Metabolism 2008;57:183-1991. 
252 19. Loder RT, and 47 coinvestigators from 33 orthopaedic centers and 6 continents. The demographics of 253 slipped capital femoral epiphysis. An international multicenter study. Clin Orthop 1996;322:8-27.

254 20. Sevenler D, Buckley MR, Kim G, van der Meulen MCH, Cohen I, Bonassar LJ. Spatial periodicity in 255 growth plate shear mechanical properties is disrupted by vitamin D deficiency. J Biomech. 2013;46:1597-1603.

256 21. Arkader A, Woon RP, Gilsanz V. Can subclinical rickets cause SCFE? A prospective pilot study. $J$

257 Pediatr Orthop 2015;35:e72-e75.

258 22. Madhuri V, Arora SK, Dutt V. Slipped capital femoral epiphysis associated with vitamin D deficiency. 259 Bone Joint J 2013;95-B:851-854.

260 23. Judd J, Welch R, Clarke A, Reading IC, Clarke NMP. Vitamin D deficiency in slipped upper femoral 261 epiphysis: time to physeal fusion. J Pediatr Orthop 2016;36:247-252.

262 24. Hägglund G, Bylander B, Hansson LI, Kärrholm J, Selvik G, Svensson K. Longitudinal growth of the 263 distal fibula in children with slipped capital femoral epiphysis. J Pediatr Orthop 1986;6:274-277.

264 25. Hägglund G, Hansson II, Ordeberg G. Epidemiology of slipped capital femoral epiphysis in southern 265 Sweden. Clin Orthop 1984;191:82-94. 


\section{LEGENDS FOR FIGURES}

270 Figure 1: The percentage of all SCFEs by month of presentation. Cosinor analysis demonstrated an excellent fit

271 using a 12 month periodicity with the equation percentage of SCFEs $=8.338+0.961(\cos (30 t-15)-226)$, where $t=1$

272 is January, 2 = February, $11=$ November, 12 = December. This was statistically significant $\left(\mathrm{r}^{2}=0.58, \mathrm{p}=0.021\right)$.

273 The peak was August 17 (arrow). The data are represented by the bars and the best fit cosinor curve by the bold

274 black line.

Figure 2: Differences in SCFE seasonal variation by latitude.

277 A: Percentage of SCFEs by month of presentation in northern latitudes. Cosinor analysis demonstrated an excellent

278 fit using a 12 month periodicity for both those between $35-40^{\circ} \mathrm{N}$ latitude (black squares and solid line) and $>40^{\circ} \mathrm{N}$

279 latitude (open triangles and dashed line). The equation for the $35-40^{\circ} \mathrm{N}$ latitude group is percentage of SCFEs $=$

$2808.338+1.029(\cos (30 \mathrm{t}-15)-225)\left(\mathrm{r}^{2}=0.55, \mathrm{p}=0.028\right)$ - peak August 16 (solid arrow); for the $>40^{\circ} \mathrm{N}$ latitude group is

281 percentage of SCFEs $=8.339+1.337(\cos (30 t-15)-235)\left(r^{2}=0.74, p=0.002\right)-$ peak August 26 (dashed arrow).

282

283 B: Percentage of SCFEs by month of presentation in the southern latitudes. Cosinor analysis demonstrated an

284 excellent fit using a 5 month periodicity for those between $31-35^{\circ} \mathrm{N}$ latitude (black triangles and solid line) with the

285 equation percentage of SCFEs $=8.441+0.856(\cos (72 t-36)-226)\left(r^{2}=0.52, p=0.036\right)$. Since the periodicity is 5

286 months, the December 31 and January 1 points are not exactly equal. The peaks were April 6 and September 5

287 (solid arrows). For those living $<31^{\circ} \mathrm{N}$ latitude (open squares and dashed line) there was no significant cosinor fit. 


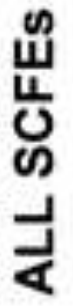

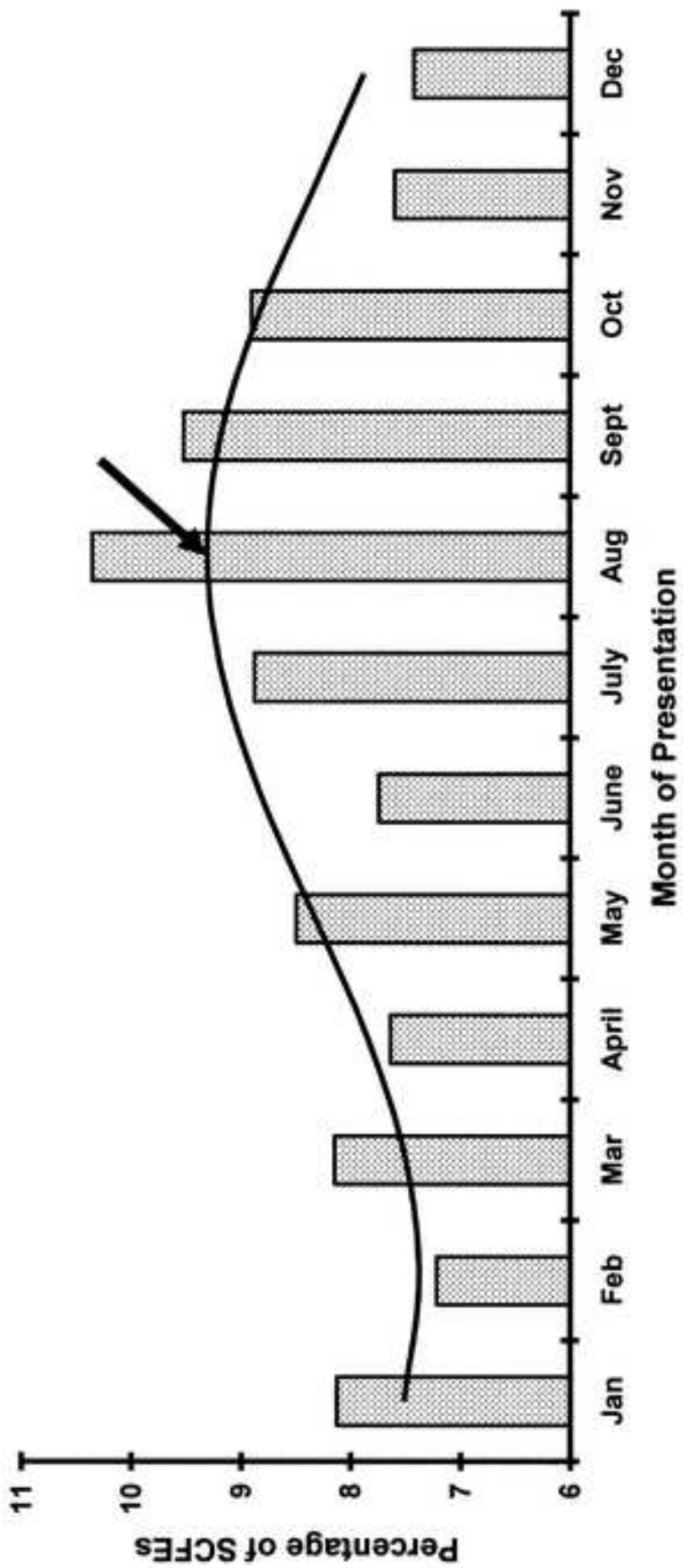




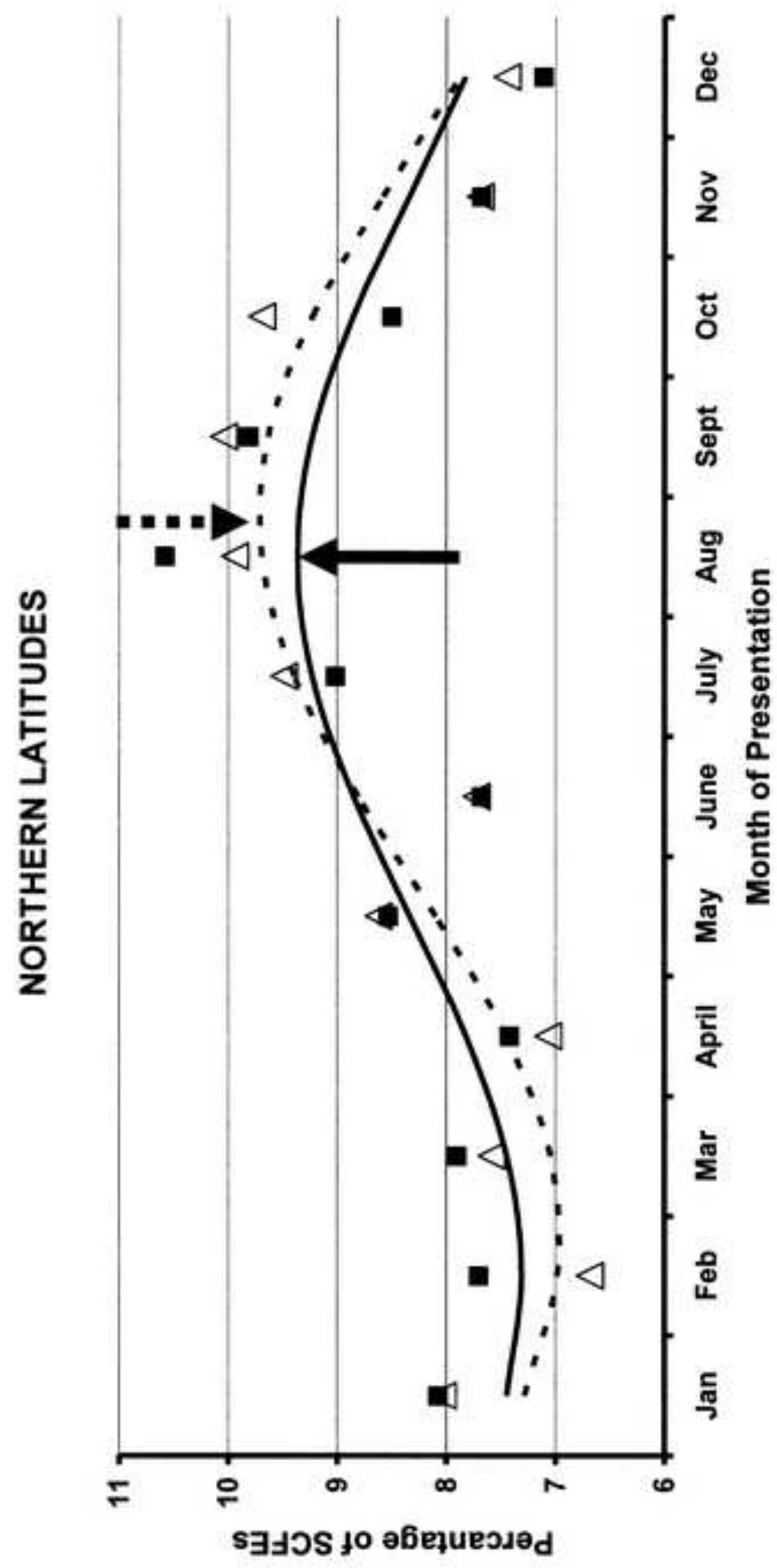




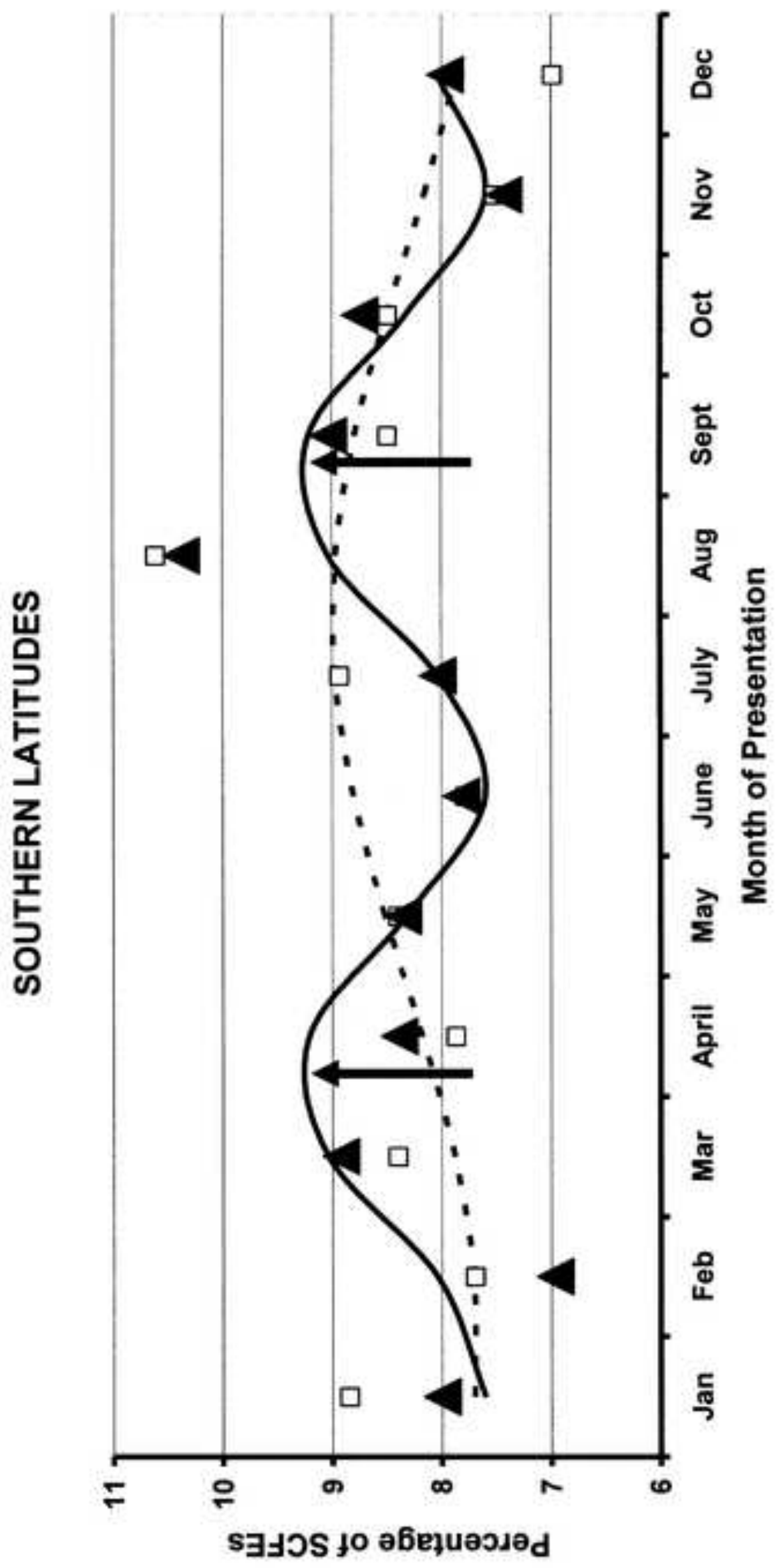


Click here to access/download

\section{Supplemental Data File (.doc, .tif, pdf, etc.) Supplemental Materials refs.doc}

